\title{
Isolation, culture, purification and ultrastructural investigation of cardiac telocytes
}

\author{
YAN-YAN LI, SONG ZHANG, YI-GANG LI and YAN WANG \\ Department of Cardiovascular Diseases, Xinhua Hospital, Shanghai Jiaotong University, \\ School of Medicine, Shanghai 200092, P.R. China
}

Received December 27, 2015; Accepted May 31, 2016

DOI: $10.3892 / \mathrm{mmr} .2016 .5386$

\begin{abstract}
Telocytes (TCs), a novel type of stromal cells, are crucial to cardiac renovation and regeneration. To dissect the pathophysiological effects of cardiac TCs in heart disease, it is essential to develop an effective method to isolate, culture, purify and characterize these cells. In the present study, cardiac TCs were isolated from the hearts of rats by enzymatic digestion. Histology and CD34/PDGFR $\alpha$ expression by flow cytometric assay were used to characterize the cultured cardiac TCs, which were purified by flow cytometric sorting and confirmed by immunofluorescence and electron microscopy. Typical TCs were observed in primary culture, with these exhibiting typical fusiform cell bodies with long moniliform telopodes. Based on flow cytometric sorting with antibodies to CD34 and PDGFR $\alpha$, there was a substantial increase in the purity of cardiac TCs. Furthermore, immunofluorescence demonstrated that almost all the sorted TCs expressed vimentin, a marker of TCs. Moreover, electron micrographs showed typical TCs based on their ultrastructural features. Using this method, we developed a reproducible protocol for the isolation and purification of cardiac TCs from rat hearts, which yielded TCs with typical characteristics.
\end{abstract}

\section{Introduction}

Telocytes (TCs) are a type of interstitial cells first identified by Popescu's group in 2005 and officially named in 2010. They exist in many tissues and organs, including those of humans and other vertebrates (1-3). TCs are characterized by a small cell body and long, slender processes called telopodes (Tps). As a distinctive feature, Tps are extremely long with thin

Correspondence to: Dr Song Zhang, Department of Cardiovascular Diseases, Xinhua Hospital, Shanghai Jiaotong University, School of Medicine, 1665 Kongjiang Road, Shanghai 200092, P.R. China E-mail: zhangsong3961@xinhuamed.com.cn

Key words: telocytes, isolation, culture, CD34/PDGFR $\alpha /$ vimentin, purification prolongations, with alternation of dilations (podoms) and thin segments (podomeres) (4-7).

In the heart, TCs have been observed in the myocardium, epicardium, endocardium, valves and cardiac stem cell niches (3-5,8-10). Based on different junction types, TCs are documented to be in close contact with virtually all types of cells in the human heart, such as cardiac stem cells, cardiomyocyte progenitors, blood capillaries, nerve endings and other cells found in the interstitial space $(9,11)$. They form the interstitial cell network and contribute to the regulation of homeostasis, and renovation and regeneration in the heart (12). Additionally, TCs have been demonstrated to be important interstitial cells to guide or nurse putative stem cells and progenitor cells in stem cell niches in a number of tissues and organs $(1,13)$. Thus, TCs may serve as a new target for regenerative medicine. Previous studies have indicated that the number of TCs reduced and the interstitial cell network was impaired during myocardial infarction $(14,15)$. However, after the transplantation of cardiac TCs in infarcted and border zones of the heart, the infarction size decreased and myocardial function was improved $(14,15)$. In addition, TCs have been found in the atrial appendages and shown to be involved in isolated atrial amyloidosis and the pathogenesis of atrial fibrillation (9).

Although increasing numbers of studies on TCs have emerged, the majority are limited to the investigation of their histology and morphology. Due to numerous difficulties in cell separation, purification and cultivation, research on their function and the mechanisms behind their biological effects progressing slowly. Therefore, the present study ultilized a novel method for the separation and purification of TCs. In addition to the distinctive morphology of TCs, their molecular markers have additionally been identified to some extent. Although TCs have no clear immunophenotypic markers, a number of studies have reported that TCs are capable of binding different antibodies $(16,17)$. Based on several reports, it has been suggested that double-positive immunostaining with cluster of differentiation (CD)34/platelet-derived growth factor receptor $\alpha$ (PDGFR $\alpha$ ) and CD34/vimentin is appropriate for identifying TCs (16). Based on this approach, the present study sorted CD34/PDGFR $\alpha$ double positive cells by flow cytometry to purify TCs, and then confirmed their identity by immunofluorescence with anti-vimentin antibodies and electron microscopy. Establishing a successful protocol will enable the large-scale isolation and culture of TCs. 


\section{Materials and methods}

Isolation and culture of TCs from heart tissues. This study was approved by the ethics committee of Xinhua Hospital Affiliated to Shanghai Jiaotong University School of Medicine (Shanghai, China; approval no. XHEC-F-2016-012). Six-week-old female Sprague Dawley (150-200 g) rats (Shanghai Songlian Laboratory Animal Farms, Production license: SCXK2007-0011) were anesthetised with 3\% pentobarbital sodium. The hearts were removed under sterile conditions and placed in $50 \mathrm{ml}$ centrifuge tube with ice-cold Dulbecco's phosphate-buffered saline (D-PBS) supplemented with $1 \%$ penicillin and streptomycin (PS). Following rinsing with fresh D-PBS to remove blood, the hearts were minced into millimeter-sized pieces in a sterile culture dish containing Dulbecco's modfied Eagle's medium (DMEM)/F12 (12400-024, Gibco; Thermo Fisher Scientific, Inc., Waltham, MA, USA) supplemented with $1 \%$ PS. The pieces were washed twice using a short centrifugation at $250 \mathrm{x} g$ for $1 \mathrm{~min}$ at room temperature and resuspended in D-PBS to remove the blood. Subsequently, an enzymatic digestion medium was added and the mixture was incubated at $37^{\circ} \mathrm{C}$ on a shaker at $180 \mathrm{rpm}$ for $40 \mathrm{~min}$. The enzymatic digestion medium was a mixture of $1.5 \mathrm{mg} / \mathrm{ml}$ collagenase type 2 (V900892; Sigma-Aldrich, St. Louis, MO, USA), DMEM/F12 and 1\% PS. The solution was filtered through a $41 \mu \mathrm{m}$ nylon mesh (EMD Millipore, Billerica, MA, USA) and the collected cell suspension was centrifuged at $300 \mathrm{x} \mathrm{g}$ for $10 \mathrm{~min}$. The cells were then seeded into sterile culture dishes containing $10 \mathrm{ml}$ DMEM/F12 supplemented with $10 \%$ fetal bovine serum (FBS; 16000-044; Gibco; Thermo Fisher Scientific, Inc.) and 1\% PS and cultured in a humidified atmosphere at $37^{\circ} \mathrm{C}$ for $1 \mathrm{~h}$ to allow fibroblast attachment. The unattached cells (containing TCs) were replated onto a new dish with the above medium, and the medium was replaced every 2 days thereafter. Cell cultures were examined using an inverted microscope and photographed at 72 and $96 \mathrm{~h}$ after seeding. The cells were detached by digestion in $0.25 \%$ trypsin/EDTA (Invitrogen; Thermo Fisher Scientific, Inc.) for 1-2 min after they had grown to $80 \%$ confluence, and then reseeded at a split ratio of 1:3 under the same conditions.

Flow cytometric analysis and sorting of isolated cardiac TCs. TC content in the enriched cultures was assessed by double labeling with phycoerythrin-conjugated monoclonal anti-CD34 (ab187284; Abcam, Cambridge, MA, USA) and rabbit polyclonal anti-PDGFR $\alpha$ (sc-338; Santa Cruz Biotechnology, Dallas, TX, USA). The TCs were analyzed using a BD FACS CantoII cytometer (BD Biosciences, San Jose, CA, USA). Passage-2 cells were washed with D-PBS and harvested by trypsinization. The samples were stained with the above antibodies for $1 \mathrm{~h}$ at room temperature. Cells were then incubated with donkey anti-rabbit H\&L-labeled secondary antibodies (ab150075; Abcam) for $30 \mathrm{~min}$ at room temperature. As a negative control, unstained cell aliquots were incubated with D-PBS under the same conditions. According to the results of the identification, the flow cytometry was adjusted to set the gate and CD34 $/{ }^{+} \mathrm{PDGFR} \alpha^{+}$ cells were sorted to purify the cardiac TCs. The sorted cells were collected in a collecting tube, which was prefilled with complete culture medium and $2 \%$ PS, and then centrifuged at
$300 \mathrm{x} \mathrm{g}$ for $5 \mathrm{~min}$. The cell density was adjusted to $1 \times 10^{5} / \mathrm{ml}$ with DMEM/F12 medium containing 10\% FBS and 1\% PS, following which the cells were seeded into sterile culture dishes and cultured in a humidified atmosphere of $5 \% \mathrm{CO}_{2}$ at $37^{\circ} \mathrm{C}$.

Immuofluorescent staining. As an independent confirmation and to visualize the appearance of sorted TCs, immunofluorescent staining was conducted on cells grown on coverslips. The sorted cells were fixed in $4 \%$ formaldehyde for $15 \mathrm{~min}$, washed three times in D-PBS and then incubated in 5\% bovine serum albumin for a further $20 \mathrm{~min}$. Subsequently, cells were incubated with rabbit monoclonal anti-vimentin antibodies (ab92547; Abcam) at $4^{\circ} \mathrm{C}$ overnight in the dark. After washing with PBS three times, cells were incubated with Alexa Fluor 594 labeled anti-rabbit secondary antibodies (8889; Cell Signaling Technology, Inc., Danvers, MA, USA) at $37^{\circ} \mathrm{C}$ for $1 \mathrm{~h}$, and then stained with 4',6-diamidino-2-phenylindole (DAPI; P36935; Thermo Fisher Scientific, Inc.). Finally, the immunolabeled samples were observed and imaged using an Olympus IX83 fluorescence inverted microscope (Olympus Corporation, Tokyo, Japan).

Transmission electron microscopy (TEM). Cell samples were processed for TEM according to routine procedures as previously described $(4,9)$. In brief, cell samples were fixed with $2 \%$ glutaraldehyde in PBS for $2 \mathrm{~h}$ at $4^{\circ} \mathrm{C}$. Following two rinses in PBS for $10 \mathrm{~min}$, the samples were postfixed in $1 \%$ phosphate-buffered osmium for $2 \mathrm{~h}$ at $4^{\circ} \mathrm{C}$. Subsequently, the samples were dehydrated in an increasing ethanol series $(30,50,70,80,95$ and $100 \%)$, cleared in propylene oxide and embedded in araldite. Ultrathin sections (50-100 nm) sections were obtained and stained with electronic lead citrate. The sections were examined with a Morgagni 286 transmission electron microscope (FEI Company, Eindhoven, Netherlands). Digital electron micrographs were recorded with a MegaView III charge-coupled device using iTEM SIS software (Olympus Soft Imaging Systems, Münster, Germany).

Statistical analysis. Data were analysed using SPSS software, version 19 (IBM SPSS, Armonk, NY, USA) and Student's two-tailed t-test. $\mathrm{P}<0.05$ was considered to indicate a statistically significant difference.

\section{Results}

Culture and purification of TCs from heart tissue. Following $72 \mathrm{~h}$ of primary culture, single adherent cells were observed in a distinct area of the culture dish. These cells were rhombic or irregular in shape, highly refractive, inconsistent in size and shape, clear at boundaries, but with obvious characteristic projections (Fig. 1A). After $96 \mathrm{~h}$ of culture, a cell monolayer was observed, with TCs exhibiting the characteristic outline of a small cell body and long moniliform Tps. Additionally, connections between the cells by Tps were observed, with these interconnected in the form of a network (Fig. 1B). TCs are characterized by a small cell body and the presence of Tps. As a distinct feature, Tps have extremely long and thin prolongations, with alternation of podoms and podomeres (Fig. 1C and D). After 7 days, the cells grew to $80 \%$ confluence in culture. Following the initial culture of cardiac 
A

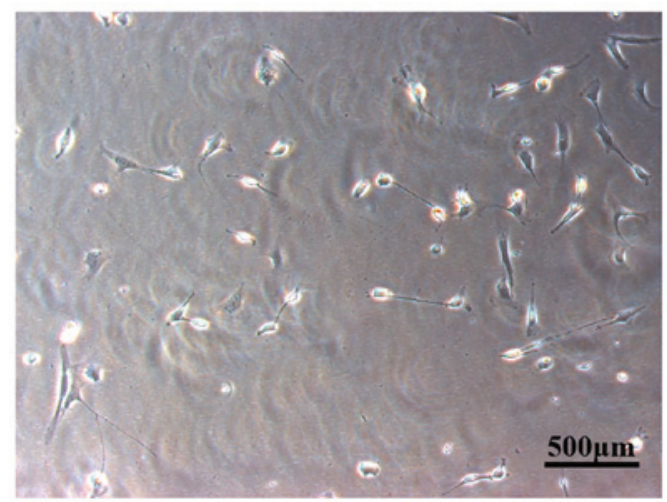

C

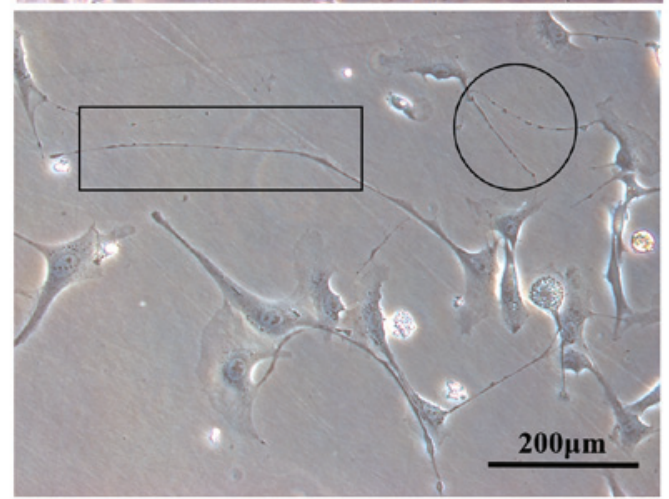

B

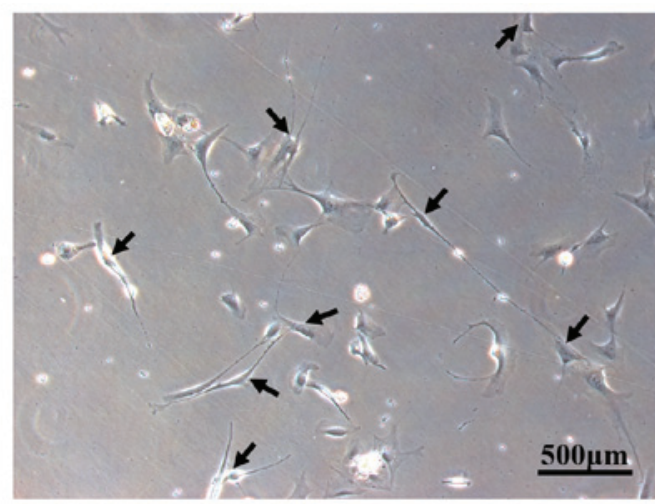

D

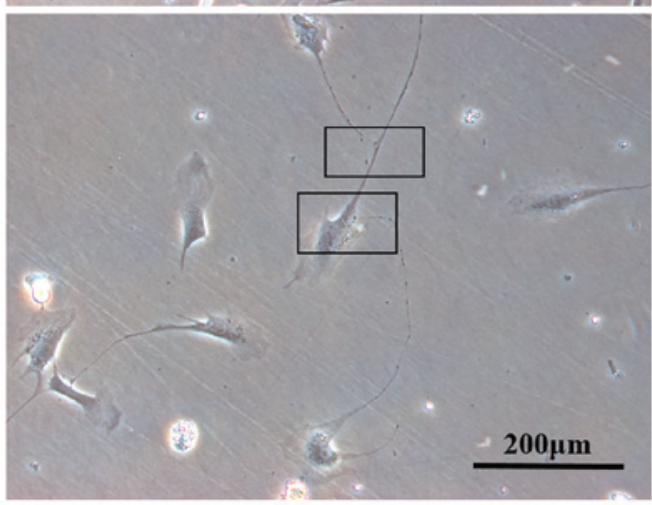

Figure 1. TCs shown under a light microscope in primary culture. (A) A few cells had adhered 72 h after isolation. (B) Arrows show typical TCs with long, thin Tps at $96 \mathrm{~h}$ in primary culture. (C) TCs with typical moniliform aspects: Alternation of podoms and podomeres (as shown in black frame). (D) TC connections were formed by Tps and the cell body. TCs, telocytes; Tps, telopodes.

A

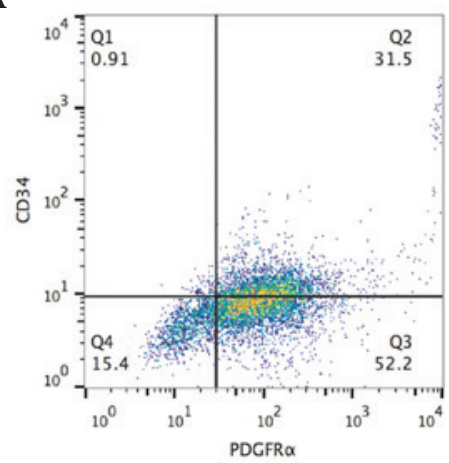

B

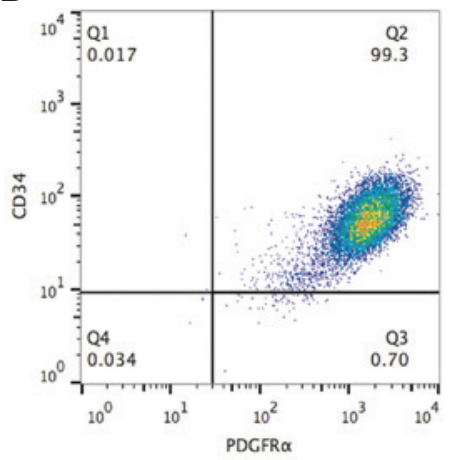

C

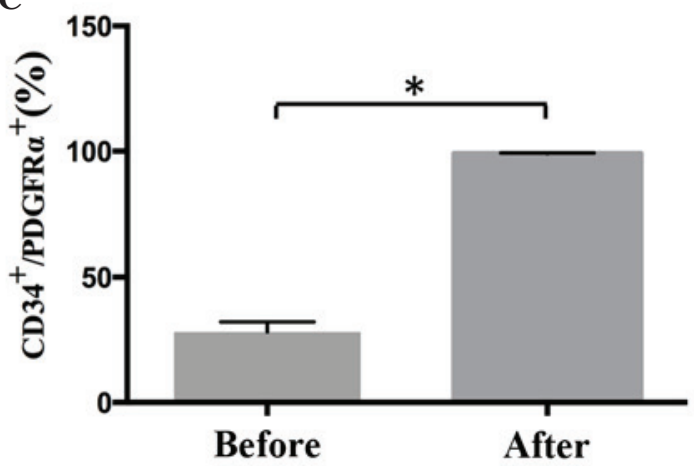

Figure 2. Flow cytometry revealed double positive expression of CD34 and PDGFR $\alpha$ (A) before and (B) after cell sorting. (C) Quantification of CD34/PDGFR $\alpha$ double positive cells. Data are presented as the mean \pm standard deviation of three independent experiments. ${ }^{*} \mathrm{P}<0.05$. CD34, cluster of differentiation 34 ; $\operatorname{PDGFR} \alpha$, platelet derived growth factor receptor $\alpha$.

primary cells, adherent cells were digested and then stained for the markers CD34 and PDGFR $\alpha$, with positive expression identified by flow cytometry (Fig. 2). The proportions of cells were as follows: CD34/PDGFR $\alpha^{-}, 15.4 \%$; $\mathrm{CD}^{-} 4^{+} / \mathrm{PDGFR} \alpha-$, $0.91 \%$; CD34-/PDGFR $\alpha+, 52.2 \%$; and CD34 ${ }^{+} / \mathrm{PDGFR}^{+}$, $31.5 \%$ (Fig. 2A). Following cell sorting, the proportion of $\mathrm{CD}^{2}{ }^{+} / \mathrm{PDGFR}^{+}$cells reached $99.3 \%$ (Fig. 2B). The sorted cells were plated and cultured under normoxic conditions.

Immunofluorescence. In order to further analyze and characterize the sorted $\mathrm{CD} 34^{+} / \mathrm{PDGFR} \alpha^{+}$cells, we examined them for immunofluorescence following incubation with anti-vimentin antibodies. Detection of immunofluorescence in the sorted cells demonstrated the presence of TCs as vimentin-positive cells, corresponding to the phenotype described previously by others either in situ $(18,19)$ or in vitro $(20)$.

The percentage of vimentin-positive cells was quantified and the mean was determined from 5 randomly selected magnification, $\mathrm{x} 200$ fields. The results showed that $96.7 \%$ of the sorted TCs were vimentin positive. The cells showed red immunofluorescence, indicating that they had been labeled with antibody and thus expressed vimentin and the nucleus was stained blue with DAPI (Fig. 3). Flow cytometric sorting and immunofluorescence of the isolated cells revealed positive expression of CD34, PDGFR $\alpha$ and vimentin, which are markers for TCs. 
A

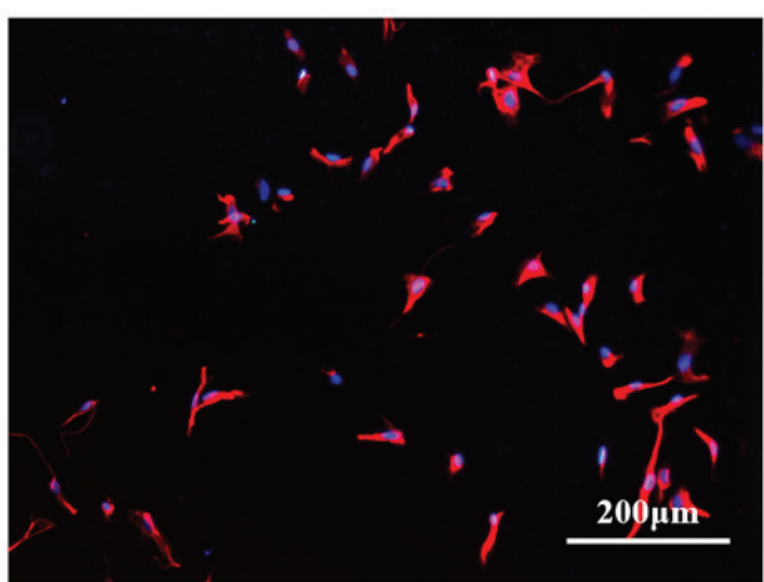

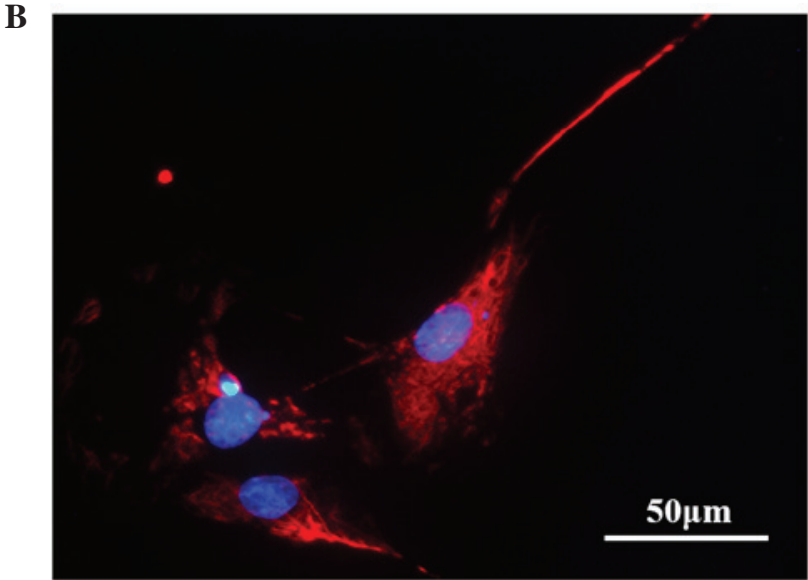

Figure 3. Immunofluorescent staining for vimentin in the sorted cardiac TCs. (A) Fluorescence inverted microscopy showed that nearly all sorted TCs are positive for vimentin (red). Nuclei were counterstained with DAPI (blue). (B) Higher magnification of the telocytes. Staining was concomitant on cell body and thin telopode. Original magnification, $\mathrm{x} 400$. TCs, telocytes.

A

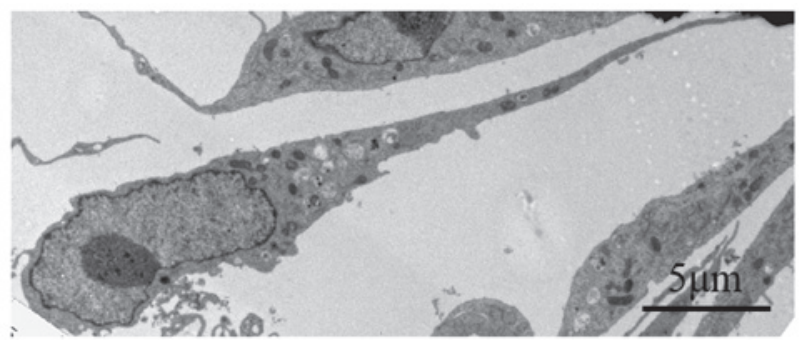

B

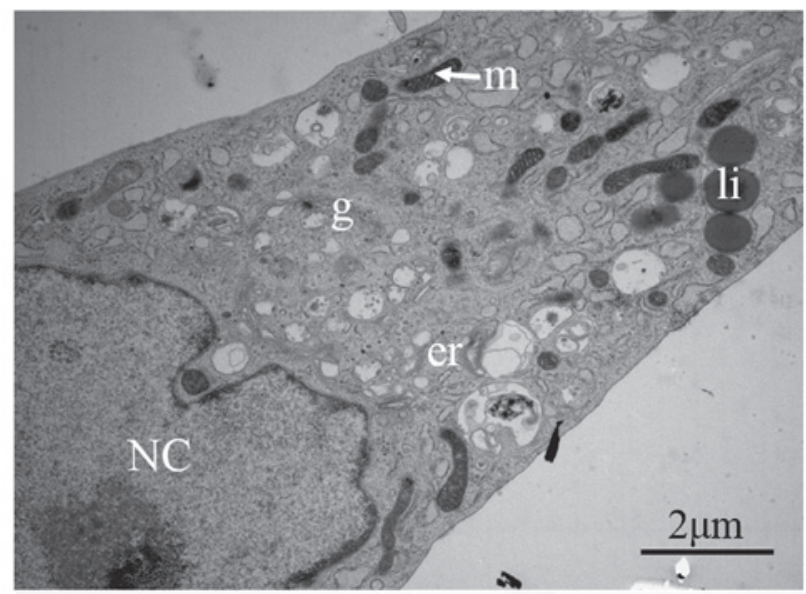

C

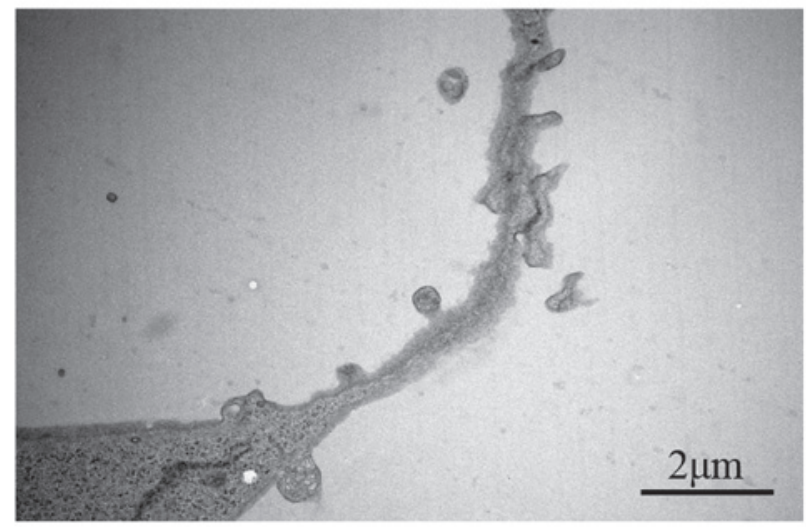

Figure 4. Transmission electron micrograph of a TC. (A) TCs had a small oval body, mostly occupied by the nucleus, encircled by a small amount of cytoplasm. (B) The structures in the cytoplasm were: $\mathrm{m}$, mitochondria; li, lipid droplets; g, Golgi apparatus; er, endoplasmic reticulum; and NC, nucleus. (C) Higher magnification of a telopode. TC, telocyte.
Transmission electron microscopy. Using electron microscopy, we noted that TCs had a small oval body, mostly occupied by the nucleus and encircled by a small amount of cytoplasm (Fig. 4A). Additionally, we observed that the cytoplasm was filled with mitochondria, lipid droplets, a small Golgi apparatus, in addition to elements of smooth and rough endoplasmic reticulum (Fig. 4B). Furthermore, higher magnification images of Tps were obtained (Fig. 4C). As one of the most striking features of TCs, the connections were organized through homocellular junctions. Figure 5A shows that tight contacts (atypical junctions) occurred between Tps and the TC cell body. Apart from the homocellular junctions, TCs have been demonstrated to serve an important role in cellular communication in the heart by releasing extracellular vesicles (EVs) (Fig. 5B-D). Different types of EVs were observed: Exosomes, mainly as intraluminal vesicles filled with multivesicular bodies (Fig. 5B); ectosomes, released from the plasma membrane of the cell body and Tps (Fig. 5C); and clusters of endomembrane vesicles encircled by the plasma membrane, termed multivesicular cargo (Fig. 5D).

\section{Discussion}

Cardiac TCs are a small shape-specific cell type in heart interstitial tissue. They can be distinguished from other cells easily because of their characteristic morphological structure in situ. As the positioning of cardiac TCs gradually becomes clearer, functional research on purified TCs has become inevitable.

In the present study, during culture it was observed that TCs exhibited the typical characteristics of a small cell body and Tps. We also found connections between TCs or between TCs and other cell types interconnected in the form of a network (Fig. 1B). Different cell types have differing adhesion abilities and require different times for adhesion in cell suspensions obtained by enzymatic digestion. In the present study, fibroblast attachment occurred within $2 \mathrm{~h}$ after seeding, while TC attachment took $24 \mathrm{~h}$ (17). However, a higher proportion of TCs could be acquired after differential adhesion for $1 \mathrm{~h}$ (data not shown). Therefore, we selected differential adhesion for $1 \mathrm{~h}$ to partially purify cardiac TCs in preliminary culture. 
A

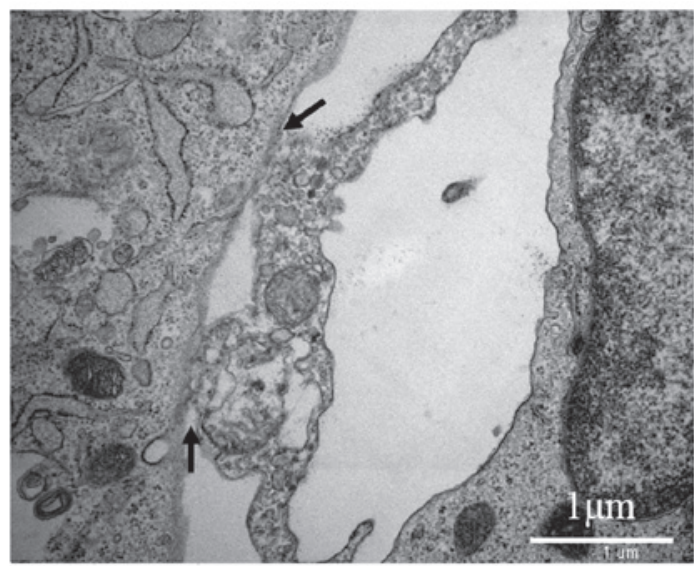

C

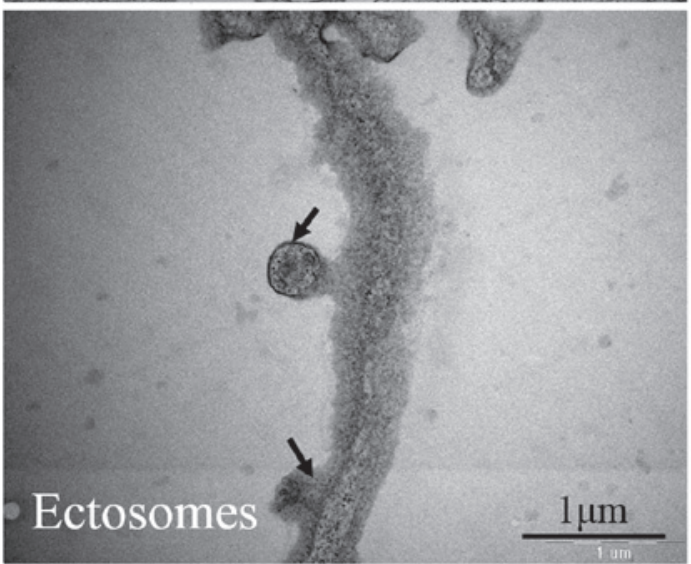

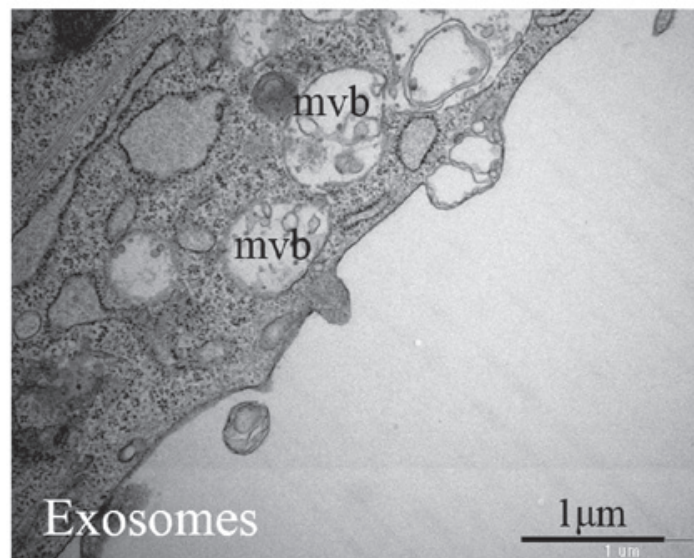

D

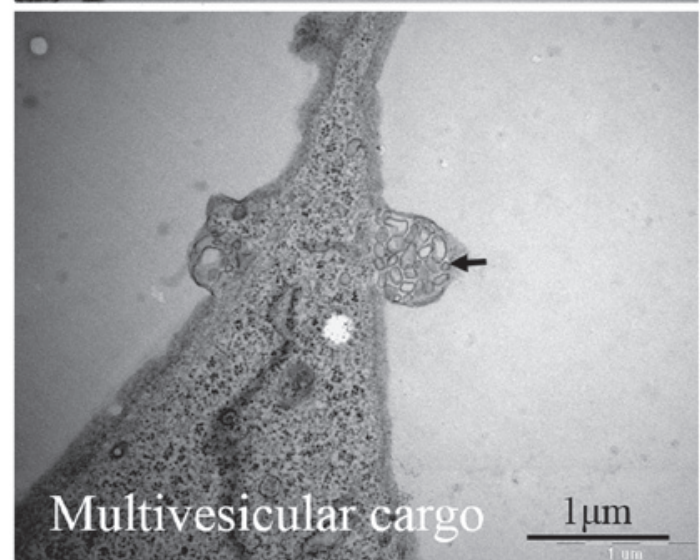

Figure 5. Transmission electron micrograph showing connections of Tp with TC. (A) Black arrows show direct contacts (atypical junctions) between Tp and TC cell body. Extracellular vesicles: (B) Exosomes, intraluminal vesicles filled with mvbs; (C) ectosomes (arrow) released from the plasma membrane of the Tps; (D) multivesicular cargo (arrow), clusters of endomembrane vesicles encircled by the plasma membrane from a Tp. Tp, telopode; TC, telocyte; mvb, multivesicular body.

Previous studies have confirmed that TCs are able to bind various antibodies $(21,22)$. Immunohistochemistry performed on a range of organs and tissues has demonstrated phenotypical traits for TCs, and a few markers have been verified to label them. The most reliable of these markers seems to be CD34 $(1,23)$. CD34 is a highly glycosylated transmembrane glycoprotein. At present, increasing numbers of studies have shown that CD34 serves an important role in mediating cell adhesion, participating in the migration and positioning of hematopoietic stem cells involved in inflammation and lymphocyte homing. Cismasiu et al (24) observed a similar biological function of TCs.

PDGFR $\alpha$ and PDGFR $\beta$ are two types of tyrosine kinases activated by PDGF, which are important in cell survival, proliferation, differentiation and migration $(25,26)$. In particular, PDGFR $\alpha$ is important during embryonic organogenesis and development. PDGFR $\alpha$ belongs to the type III receptor tyrosine kinase family and is an angiogenic factor (27). Recently, CD34/PDGFR $\alpha$ have been proposed to be specific markers for cardiac TCs (14). Zhou et al (28) reported that PDGFR $\alpha$ positive cells accounted for a large proportion of rat cardiac TC-enriched cultures, and one-third of all cells were CD34/PDGFR $\alpha$ double positive. The results of the present study confirmed the positive expression of PDGFR $\alpha$, consistent with previous studies. Therefore, we selected CD34 and PDGFR $\alpha$ as the markers to identify and purify cardiac TCs.
Currently available cell isolation techniques are predominantly based on properties including antibody binding, particle size, density gradient, adherence or absorbance (29-31). Above all, antibody-binding methodology depends on antigen-antibody binding of cell surface biomarkers, and hence provides precise sorting, with techniques such as fluorescence-activated cell sorting (FACS) and magnetic-activated cell sorting (MACS) (32-34). However, the problem with MACS is that the remaining magnetic beads on sorted cells affect further culture and testing. Density gradient centrifugation is relatively simple, however, the purity is relatively low. Conversely, the different media used for separation risk cell damage. Therefore, we used FACS to sort cardiac TCs in the present study. Our findings for double positive expression of CD34 and PDGFR $\alpha$ before and after flow cytometry sorting were 31.5 and $99.3 \%$, respectively, which demonstrates high efficiency.

On the basis of FACS, immunofluorescent staining for vimentin, another TC marker, was used to confirm the sorted TCs (2). Of the sorted TCs, $96.7 \%$ were vimentin positive. This contributed to the aim of confirming cardiac TCs by three markers and was consistent with the results of immunohistochemistry to localize cardiac TCs, which co-expressed PDGFR $\alpha$, CD 34 and vimentin.

As a method to identify TCs, TEM offered high-resolution information on ultrastructural features, which aids in understanding theirfunction. Though the morphologicalcharacteristics 
of TCs in culture were not completely in accordance with the characteristics observed in vivo, the main appearance was quite consistent $(35,36)$. The distinctive morphology of Tps is presented in Fig. 4. As TEM is a 2D assay of an ultrathin section ( $\sim 60 \mathrm{~nm}$ ), the number of prolongations differs depending on the position and angle of sectioning. Additionally, as the microenvironment in culture is different to that in a normal heart in vivo, it is understandable that the appearance of TCs in culture was not fully typical of their morphology in vivo.

A large number of TCs are located around the stem pool, which exists in various organs and tissues and is crucial to the survival, migration and differentiation of stem cells, particularly during tissue renewal (37). Studies confirm that TCs form a network in the myocardial interstitium and play an important role in short- and long-distance intercellular communication by TC-TC junctions, in addition to heterocellular junctions of TCs (11). The present study additionally showed that tight contacts (atypical junctions) occurred between the Tps and the TC cell body (Fig. 5B). Furthermore, Tps wrap around stem cells to supply nutrition or contribute to signal transduction. Therefore, TCs are also called stem cell accessory cells (38). Previous studies have reported that intercellular connections are formed between TCs and stem cells in the stem cell pool that participates in signal transduction by these cells $(11,39,40)$. In addition, TCs can secrete extracellular vesicles in different forms, such as exosomes, ectosomes and multivesicular cargo (Fig. 5B-D). These vesicles may contain small molecules or macromolecular signaling proteins functioning as intercellular shuttles for the communication of biological signals, which are critical to the development and differentiation of stem cells and the collection of other accessory cells from the blood to the stem cell pool (16). Thus, there is emerging evidence that TCs and stem cells may act in tandem (16). A promising idea is to use stem cells assisted by TCs to help repair damage and necrosis in future cell-based cardiac repair strategies. Based on our hypothesis, it is of great importance to purify cardiac TCs and the present study contributes to that effort.

In summary, the method used in the present study is relatively simple and effective. Furthermore, this study demonstrates a new purification strategy for purifying TCs by flow cytometric sorting with antibodies against CD34 and PDGFR $\alpha$. The purified cardiac TCs may become an important tool for elucidating their biological effects, in particular in vitro studies. We believe that this method will contribute to providing novel approaches to understanding the regulation of homeostasis, renovation and regeneration in the heart.

\section{Acknowledgements}

The present study was supported by the Nature Science Foundation of China (grant no. 81170124/H0203).

\section{References}

1. Popescu LM and Faussone-Pellegrini MS: TELOCYTES - a case of serendipity: The winding way from Interstitial Cells of Cajal (ICC), via Interstitial Cajal-Like Cells (ICLC) to TELOCYTES. J Cell Mol Med 14: 729-740, 2010.

2. Suciu L, Popescu LM, Gherghiceanu M, Regalia T, Nicolescu MI, Hinescu ME and Faussone-Pellegrini MS: Telocytes in human term placenta: Morphology and phenotype. Cells Tissues Organs 192: 325-339, 2010
3. Kostin S: Myocardial telocytes: A specific new cellular entity. J Cell Mol Med 14: 1917-1921, 2010.

4. Popescu LM, Manole CG, Gherghiceanu M, Ardelean A, Nicolescu MI, Hinescu ME and Kostin S: Telocytes in human epicardium. J Cell Mol Med 14: 2085-2093, 2010.

5. Gherghiceanu M, Manole CG and Popescu LM: Telocytes in endocardium: Electron microscope evidence. J Cell Mol Med 14: 2330-2334, 2010

6. Rusu MC, Jianu AM, Mirancea N, Didilescu AC, Mănoiu VS and Păduraru D: Tracheal telocytes. J Cell Mol Med 16: 401-405, 2012.

7. Zheng Y, Zhu T, Lin M, Wu D and Wang X: Telocytes in the urinary system. J Tranl Med 10: 188, 2012.

8. Faussone-Pellegrini MS and Bani D: Relationships between telocytes and cardiomyocytes during pre- and post-natal life. J Cell Mol Med 14: 1061-1063, 2010.

9. Mandache E, Gherghiceanu M, Macarie C, Kostin S and Popescu LM: Telocytes in human isolated atrial amyloidosis: Ultrastructural remodelling. J Cell Mol Med 14: 2739-2747, 2010.

10. Yang Y, Sun W, Wu SM, Xiao J and Kong X: Telocytes in human heart valves. J Cell Mol Med 18: 759-765, 2014.

11. Gherghiceanu M and Popescu LM: Cardiac telocytes-their junctions and functional implications. Cell Tissue Res 348: 265-279, 2012.

12. Barile L, Lionetti V, Cervio E, Matteucci M, Gherghiceanu M, Popescu LM, Torre T, Siclari F, Moccetti T and Vassalli G: Extracellular vesicles from human cardiac progenitor cells inhibit cardiomyocyte apoptosis and improve cardiac function after myocardial infarction. Cardiovasc Res 103: 530-541, 2014.

13. Gherghiceanu M and Popescu LM: Cardiomyocyte precursors and telocytes in epicardial stem cell niche: Electron microscope images. J Cell Mol Med 14: 871-877, 2010.

14. Zhao B, Chen S, Liu J, Yuan Z, Qi X, Qin J, Zheng X, Shen X, $\mathrm{Yu} \mathrm{Y}$, Qnin TJ, et al: Cardiac telocytes were decreased during myocardial infarction and their therapeutic effects for ischaemic heart in rat. J Cell Mol Med 17: 123-133, 2013.

15. Zhao B, Liao Z, Chen S, Yuan Z, Yilin C, Lee KK, Qi X, Shen X, Zheng X, Quinn T and Cai D: Intramyocardial transplantation of cardiac telocytes decreases myocardial infarction and improves post-infarcted cardiac function in rats. J Cell Mol Med 18: 780-789, 2014.

16. Cretoiu SM and Popescu LM: Telocytes revisited. Biomo Concepts 5: 353-369, 2014.

17. Bei Y, Zhou Q, Fu S, Lv D, Chen P, Chen Y, Wang F and Xiao J: Cardiac telocytes and fibroblasts in primary culture: Different morphologies and immunophenotypes. PLoS One 10: e0115991, 2015.

18. Vannucchi MG, Traini C, Manetti M, Ibba-Manneschi L and Faussone-Pellegrini MS: Telocytes express PDGFR $\alpha$ in the human gastrointestinal tract. J Cell Mol Med 17: 1099-1108, 2013.

19. Milia AF, Ruffo M, Manetti M, Rosa I, Conte D, Fazi M, Messerini L and Ibba-Manneschi L: Telocytes in Crohn's disease. J Cell Mol Med 17: 1525-1536, 2013.

20. Mou Y, Wang Y, Li J, Lü S, Duan C, Du Z, Yang G, Chen W, Zhao S, Zhou J and Wang C: Immunohistochemical characterization and functional identification of mammary gland telocytes in the self-assembly of reconstituted breast cancer tissue in vitro. J Cell Mol Med 17: 65-75, 2013.

21. Gangenahalli GU, Singh VK, Verma YK, Gupta P, Sharma RK, Chandra R and Luthra PM: Hematopoietic stem cell antigen CD34: role in adhesion or homing. Stem Cells Dev 15:c305-c313, 2006.

22. Kim JH, Choi SC, Park CY, Park JH, Choi JH, Joo HJ, Hong SJ and Lim DS: Transplantation of immortalized CD34 ${ }^{+}$and CD34 adipose-derived stem cells improve cardiac function and mitigate systemic pro-inflammatory responses. PLoS One 11: e0147853, 2016.

23. Vannucchi MG, Traini C, Guasti D, Del Popolo G and Faussone-Pellegrini MS: Telocytes subtypes in human urinary bladder. J Cell Mol Med 18: 2000-2008, 2014.

24. Cismaşiu VB and Popescu LM: Telocytes transfer extracellular vesicles loaded with microRNAs to stem cells. J Cell Mol Med 19: 351-358, 2015.

25. Andrae J, Gallini R and Betsholtz C: Role of platelet-derived growth factors in physiology and medicine. Genes Dev 22: 1276-1312, 2008.

26. Tallquist MD and Soriano P: Cell autonomous requirement for PDGFRalpha in populations of cranial and cardiac neural crest cells. Development 130: 507-518, 2003. 
27. Heinrich MC, Corless CL, Duensing A, McGreevey L, Chen CJ, Joseph N, Singer S, Griffith DJ, Haley A, Town A, et al: PDGFRA activating mutations in gastrointestinal stromal tumors. Science 299: 708-710, 2003.

28. Zhou Q, Wei L, Zhong C, Fu S, Bei Y, Huică RI, Wang F and Xiao J: Cardiac telocytes are double positive for CD34/PDGFR- $\alpha$. J Cell Mol Med 19: 2036-2042, 2015.

29. Chen Y, Li P, Huang PH, Xie Y, Mai JD, Wang L, Nguyen NT and Huang TJ: Rare cell isolation and analysis in microfluidics. Lab Chip 14: 626-645, 2014.

30. Spangrude GJ, Heimfeld S and Weissman IL: Purification and characterization of mouse hematopoietic stem cells. Science 241: 58-62, 1988

31. Tomlinson MJ, Tomlinson S, Yang XB and Kirkham J: Cell separation: Terminology and practical considerations. J Tissue Eng 4: $2041731412472690,2013$.

32. Schmitz B, Radbruch A, Kümmel T, Wickenhauser C, Korb H Hansmann ML, Thiele J and Fischer R: Magnetic activated cell sorting (MACS)-a new immunomagnetic method for megakaryocytic cell isolation: Comparison of different separation techniques. Eur J Haematol 52: 267-275, 1994.

33. Grützkau A and Radbruch A: Small but mighty: How the MACS-technology based on nanosized superparamagnetic particles has helped to analyze the immune system within the last 20 years. Cytometry A 77: 643-647, 2010.
34. Wilkerson MJ: Principles and applications of flow cytometry and cell sorting in companion animal medicine. Vet Clin North Am Small Anim Pract 42: 53-71, 2012.

35. Suciu L, Nicolescu MI and Popescu LM: Cardiac telocytes: Serial dynamic images in cell culture. J Cell Mol Med 14: 2687-2692, 2010.

36. Zhou J, Wang Y, Zhu P, Sun H, Mou Y, Duan C, Yao A, Lv S and Wang C: Distribution and characteristics of telocytes as nurse cells in the architectural organization of engineered heart tissues. Sci China Life Sci 57: 241-247, 2014.

37. Galiger C, Kostin S, Golec A, Ahlbrecht K, Becker S, Gherghiceanu M, Popescu LM, Morty RE, Seeger W and Voswinckel R: Phenotypical and ultrastructural features of Oct4-positive cells in the adult mouse lung. J Cell Mol Med 18: 1321-1333, 2014.

38. Kostin S and Popescu LM: A distinct type of cell in myocardium: Interstitial Cajal-like cells (ICLCs). J Cell Mol Med 13: 295-308, 2009.

39. Cretoiu D, Hummel E, Zimmermann H, Gherghiceanu M and Popescu LM: Human cardiac telocytes: 3D imaging by FIB-SEM tomography. J Cell Mol Med 18: 2157-2164, 2014.

40. Fertig ET, Gherghiceanu M and Popescu LM: Extracellular vesicles release by cardiac telocytes: Electron microscopy and electron tomography. J Cell Mol Med 18: 1938-1943, 2014. 